\title{
Genève, Dix Ans Après
}

\author{
par Fabio Padoa *
}

1. L'Association de Genève célèbre cette année le dixième anniversaire de sa fondation. C'est en effet le 20 juin 1973 que se rassemblent en Suisse ses premiers associés pour approuver ses statuts. Cette première Assemblée couronnait une série de réunions du Comité fondateur dont faisaient partie Emil FREY, Georges MARTIN, Ernst MEYER (qui devait décéder prématurément), Bernard PAGEZY, Heinz VISCHER ainsi que l'auteur de ces lignes.

Nous partagions alors le sentiment que l'assurance, nourrie aux sources du droit et des mathématiques, ne connaissait pas un rapport aussi étroit avec les sciences de l'économie dont les développements étaient pourtant essentiels à la compréhension du milieu dans lequel elle était appelée à opérer. Il nous semblait qu'à un besoin d'approfondissement de la culture économique, non pas de tel ou tel assureur, mais bien de la profession dans son ensemble, correspondait une étrange indifférence du monde économique en général, de la théorie économique et même de l'opinion politique à l'égard d'une activité telle que la nôtre qui avait pourtant son mot à dire - et parfois ses solutions à proposer - sur bien des problèmes contemporains. Il nous semblait même que l'avenir de nos entreprises était lié bien plus à l'évolution de facteurs macroéconomiques agissant autour d'elles qu'aux problèmes de gestion qui représentaient la tâche quotidienne de nos collaborateurs. Nous sentions en somme très vivement - et presque comme une condition de survie pour l'assurance libre - qu'il devenait chaque jour plus nécessaire d'en savoir plus sur les grandes orientations du milieu économique dans lequel nous opérions comme assureurs, et que d'autre part ce monde avait quelque chose à apprendre de notre expérience en matière de gestion du risque.

C'est ainsi que nous résolûmes de rassembler nos efforts pour créer une structure de recherche économique en matière de risque et d'assurance, que nous la voulûmes internationale pour placer les résultats de ses travaux au-dessus des contingences de chacun de nos marchés en particulier, et que nous décidâmes de l'implanter à Genève, haut lieu des organisations interétatiques et de l'information. Trieste.

* Président de l'Association de Genève, Administrateur de "Assicurazioni Generali », 
2. Dix années se sont écoulées depuis.

Le temps a-t-il confirmé nos intuitions d'alors ? Quels éléments nouveaux sont-ils venus s'ajouter au tableau que nous nous tracions? Quels changements se sont-ils produits ?

Telle que tout organisme vivant, l'assurance n'évolue qu'insensiblement d'un jour à l'autre ; à certains elle paraît immuable et presque figée. Et pourtant, photographiée par intervalles, elle révèle des transformations remarquables et vitales. Tout n'est pas d'affirmer que nous vivons en une époque de changement, encore faut-il discerner en nous et autour de nous ce qui change, et dans quel sens, si nous voulons saisir la direction du mouvement et nous y préparer.

Au cours des dix années qui nous séparent de la fondation de notre Association, qu'est-ce qui a changé dans l'assurance mondiale et dans quel sens ? C'est la question à laquelle je vais essayer de répondre.

D'abord, une remarque sur les dimensions : dans chacun de nos pays et même au-delà, dans chacun des pays de l'OCDE presque sans exception, la croissance du montant global des primes a dépassé celle du produit national brut. La demande d'assurance a donc augmenté plus que proportionnellement à celle de l'activité économique en général et cela aussi bien au cours des années de « boom » qu'au cours des années creuses.

Durant la même décennie, les dimensions relatives des grands espaces économiques mondiaux ont également évolué : l'Amérique du Nord a diminué sa part de l'assurance mondiale de $57 \%$ à $47 \%$, l'Europe Occidentale et le Japon l'ont augmentée respectivement de $27 \%$ à $34 \%$ et de $9 \%$ à $11 \%$. Aujourd'hui comme alors, aucun des autres espaces économiques ne dépasse $2 \%$ de l'assurance mondiale. Ces quelques pourcentages et leur évolution suffisent à confirmer que la demande d'assurance augmente en fonction de l'évolution de l'économie, donc de l'importance croissante qu'y prennent successivement le secteur industriel et celui des services. L'assurance se développe la où se développe l'industrie et trouve son terrain le plus fertile dans une société post-industrielle.

\section{Pourquoi en est-il ainsi ?}

Il appartient probablement au sociologue plus encore qu'à l'économiste d'indiquer les raisons pour lesquelles le sentiment d'insécurité domine chez l'homme dans les sociétés avancées. Ce qui est indéniable, c'est que les mécanismes collectifs mis en œuvre pour assurer à tous, du berceau à la tombe, ce qu'on appelle la sécurité sociale, ne parviennent pas à donner à leurs bénéficiaires la tranquillité promise. Ceux-ci se sentent demeurer prisonniers de structures collectives anonymes et imperscrutables sur lesquelles ils n'ont aucune prise. De même, l'entreprise industrielle ou commerciale se révèle d'autant plus vulnérable que ses dimensions augmentent et qu'elle se sert de technologies d'avant-garde, aussi fragiles que sophistiquées. L'énorme concentration de richesse en peu d'espace, l'interdépendance extrême des structures, chacune d'elles tributaire des autres, font d'une société industrielle avancée un perpétuel miracle d'équilibre et d'accord tacite, à la merci du hazard ou de la malveillance, où tout incident peut prendre les proportions d'une catastrophe. A ces conditions objectives, s'ajoute la nouvelle sensibilité sociale envers l'environnement en général ou envers tout usager, qui rend responsable quiconque exerce quelque activité, des dommages même 
non fautifs qu'il peut procurer à d'autres. Il n'est pas étonnant qu'une constellation de ce genre exalte cette demande de sécurité, donc d'assurance, que les chiffres nous ont documentée tout à l'heure pour les pays avancés.

Non sans rapport avec la prolifération des mesures de protection sociale et avec l'extension de l'emprise de l'Etat sur l'économie, l'accroissement des dépenses et de l'endettement public est à l'origine de l'inflation qui sévit en plus grande ou en moindre mesure dans toutes les économies occidentales depuis 1973 et dont le renchérissement du pétrole n'a été qu'une des causes, pour ne pas dire des prétextes. Il en dérive pour l'assurance des conséquences majeures, dont les effets différents se feront sentir encore pour longtemps sur nos comptes d'exploitation. L'élévation des taux d'intérêt tend à reléguer au deuxième rang l'importance des résultats techniques, en donnant l'illusion que leur déficit peut être comblé sans problèmes par les bénéfices que procure le maniement des fonds. L'illusion, dis-je, car la course inconsidérée à la prime accumule dans les réserves techniques un manque invisible d'abord, mais progressivement croissant, qui se révélera dans toute sa gravité le jour où, le loyer de l'argent se normalisant, les investissements ne fourniront plus les moyens pour pallier aux insuffisances des réserves. A ce moment, remonter la pente des taux de prime adéquats ne sera pas aussi facile que l'avait été la descente et les assureurs imprévoyants se trouveront en de sérieuses difficultés. Dans le même sens, l'attrait d'une activité sans problèmes de liquidité dans une période d'argent cher fait proliférer autour de l'assurance une catégorie d'opérateurs improvisés n'y voyant que l'occasion d'une spéculation à court terme, donc d'un "cash-flow underwriting » sans scrupules. Et cela aussi bien dans l'assurance en régime de liberté de prestation telle qu'elle se pratique déjà sur certains marchés internationaux que dans le domaine de la réassurance qui, elle aussi, ne demande pas de frais majeurs d'installation et semble donc une porte largement ouverte à tout venant sur l'aubaine des primes faciles.

4. C'est ainsi que si la demande d'assurance augmente comme nous l'avons vu tout à l'heure, l'offre augmente elle aussi, et de façon plus soudaine et artificielle. Aussi bien l'accroissement de la demande que celui de l'offre sont l'effet sur l'assurance de changements qui se produisent au niveau macro-économique et qu'il importe d'interpréter et même de prévoir dans leurs déroulements successifs pour y adapter la conduite de l'entreprise.

Une conséquence d'un signe différent des difficultés que rencontre le secteur public dans la gestion de l'assurance sociale, c'est par contre la nouvelle ouverture qui paraît se dessiner pour l'assurance libre du fait des déficits, partout manifestes maintenant, de la sécurité sociale. L'incapacité du régime de répartition de faire front à ses nécessités croissantes à cause de la natalité réduite et de la récession, remet en question l'ambitieux programme qui consistait à promettre tout à tous sans contrôle des dépenses ni effort personnel des bénéficiaires; on songe à nouveau à limiter les avantages de la répartition à une tranche sociale déterminée au niveau des besoins réels, et à revenir progressivement pour le reste au régime de l'accumulation, donc à l'assurance.

Une troisième conséquence de l'évolution des marchés financiers au cours de la décennie qui nous occupe, c'est la multiplication des rapports réciproques entre l'assurance et les autres institutions financières. En matière d'épargne-assurance, la monnaie 
devenant de moins en moins fidèle à sa fonction de mètre et de réservoir des valeurs, on voit les assurances sur la vie exprimer leurs recettes et leurs engagements en quotesparts de fonds d'investissement contenant des titres : actions ou obligations. Réciproquement, les banques offrent à leurs guichets certains services d'assurance à côté du service bancaire. De plus en plus, les réseaux d'intermédiaires proposent au public qu'ils démarchent le choix entre plusieurs options de placement : police d'assurance, fonds d'investissement, épargne à la construction, et ainsi de suite. C'est-à-dire que, sous la pression de l'augmentation des frais qui pèse sur toutes les institutions financières en raison d'une augmentation des salaires généralement supérieure au niveau de l'inflation, chacune d'elles cherche à les répartir sur une plus grande surface de produits et, pour ce faire, empiète sur le terrain de l'autre. La lutte de concurrence ne s'exerce plus seulement à l'intérieur du secteur des assurances, mais entre celui-ci et les secteurs contigus, qu'il y a donc lieu de mieux connaître et de mieux comprendre.

5. La nouvelle conscience de la vulnérabilité des grands ensembles industriels développe au cours des années que nous considérons ici une nouvelle spécialisation dans la gestion de l'entreprise : le « risk management». Il s'agit d'analyser point par point tous les périls assurables ou non - auxquels l'entreprise est exposée, d'en calculer les coûts et d'en prévoir les conséquences. Ce faisant, le « risk manager » est nécessairement conduit à se demander si l'assurance classique est bien la forme la plus économique de couverture qu'il soit, ou si les dimensions des biens à protéger ne permettent pas de trouver à meilleur compte un équilibre automatique à l'intérieur même du bilan général, c'est-à-dire de devenir son propre assureur. De là à former dans ce bilan un fonds spécial de compensation et plus tard à le rendre autonome sous forme d'une société dite " captive » il n'y a qu'un pas. Les avantages fiscaux aidant, les «captives » ont proliféré d'une façon massive au cours de la décennie et on en compte aujourd'hui près de 2000 . Certains estiment entre $25 \%$ et $40 \%$ du marché des risques industriels la proportion de ceux qui seront l'apanage des «captives » dans les années 1990.

6. Cette évolution, dont nous venons de repérer les causes parmi les tendances de fond du milieu économique et social qui entoure l'assurance, se manifeste également par le changement des structures propres à celle-ci. C'est ainsi que, dans l'entreprise d'assurance, à l'articulation horizontale par branches répondant à la fois à une succession historique et à un souci de précision technique, tend à succéder une répartition verticale entre risques industriels et commerciaux d'une part et risques de masse de l'autre : la qualité de la demande prend le pas sur celle de l'offre, et l'entreprise s'organise en conséquence.

L'envers commercial de cette évolution apparaît dans la concentration des grandes maisons de courtage, qui adoptent une structure internationale, se font coter en Bourse et infléchissent à leur avantage les orientations de la législation communautaire. La puissante corporation des courtiers trouve un terrain d'élection dans certains phénomènes que nous avons indiqués, tels que la multiplication de la capacité et la prolifération des « captives», qu'elle contribue à encourager. La naissance de nouveaux marchés des risques tels que le New York Insurance Exchange va dans le même sens et répond au même besoin.

Par contre les nouveaux instruments offerts par les progrès de l'informatique promettent des modes de diffusion directe de formules d'assurance pour le grand public 
et donneront sous peu à l'usager l'accès direct à l'élaborateur central de la compagnie en court-circuitant tout intermédiaire.

Dès maintenant, l'évolution de l'informatique met sur le marché de l'assurance-vie toute une catégorie de nouvelles formules flexibles, qui permettent d'adapter chaque police individuelle soit aux exigences variables de l'assuré, soit aux résultats réalisés chaque année par la gestion financière de l'entreprise. Enfin, au niveau du "management », des modèles économétriques de plus en plus raffinés introduisent de nouveaux éléments de rationalité et de planning stratégique dans la gestion foncièrement intuitive de l'entreprise d'il y a quelques années.

Au niveau micro-économique cette fois-ci, des changements importants se sont donc manifestés au cours de la décennie, induits eux aussi par des mutations qualitatives dans les possibilités mises à la disposition de la gestion par les progrès de la technologie. Il n'est point besoin de rappeler par ailleurs que ceux-ci ouvrent à l'assurance de nouveaux champs d'activité, de la protection contre les dommages provoqués par l'informatique - une branche qui au début des années 1990 sera comparable par importance à la branche incendie - à la couverture des satellites.

7. La quantité étant généralement ennemie de la qualité, l'accroissement spectaculaire de l'offre d'assurance est allée parfois aux dépens des standards de sécurité proposés. Quelqu'un a déjà parlé de "capacité apparente» du marché mondial. Certains épisodes de laisser-aller professionnel dénoncés au cœur d'institutions considérées jadis comme les sanctuaires de la correction professionnelle ont éveillé l'attention sur la nécessité de contrôles plus sévères sur les agissements des courtiers et sur la pratique de la réassurance. Il paraît inévitable que celle-ci soit soumise à son tour, comme l'ont été toutes les branches d'assurance directe par les directives de la Communauté européenne, à la surveillance d'autorités de contrôle nationales ou internationales. Il ne s'agit pas seulement de recueillir des informations et de les coordonner, mais aussi probablement de rechercher de nouvelles formules synthétiques semblables à celles de la marge de solvabilité, permettant de juger sur quelques indices comptables la qualité de la "security 》 d'un réassureur. La tendance ne semble certainement pas aller dans le sens de la "deregulation» que souhaitent certains milieux américains, mais plutôt vers une extension du contrôle. C'est le rôle de la recherche économique appliquée que de trouver des indices aptes à porter un jugement valable sur la qualité d'une entreprise sans trop en alourdir la gestion.

8. Comme on vient de le constater, l'espace qui s'ouvre à la recherche dans le domaine de l'assurance s'est remarquablement amplifié depuis dix ans. A cet élargissement du champ d'action de l'économie de l'assurance sur le plan technique s'ajoute, pour la théorie économique, la nouvelle attention consacrée aux problèmes de risque. L'incertitude radicale qui caractérise notre époque ne se manifeste pas seulement dans le domaine de la psychologie ou dans celui des décisions du chef d'entreprise - elle correspond à une attitude fondamentale de la pensée scientifique contemporaine. Les physiciens d'aujourd'hui ont mis à la place de l'univers de Newton, gouverné par les lois exactes de la gravitation, un monde où, depuis Einstein règne la probabilité et depuis Heisenberg l'indétermination : c'est le monde de l'incertitude. A l'orgueil positiviste du XIX $\mathrm{XI}^{\mathrm{e}}$ siècle ont succédé le doute mais aussi la disponibilité à l'accueil de la science d'aujourd'hui. Entraînés par le même mouvement, certains économistes avertis 
reconnaissent dans l'idée d'incertitude la variable dont leurs prédécesseurs, croyant dans un monde exact et prévisible, n'ont pas su assez tenir compte; c'est de cette façon qu'ils expliquent la défaillance de l'économie classique, incapable de trouver remède aux maux de notre époque : chômage et inflation monétaire. Le risque devient à leurs yeux un élément important pour une reconstruction révisée du cadre économique. L'assurance, qui représente avant tout une technique de l'évaluation et de la gestion du risque, acquiert ainsi la dignité d'un instrument de l'analyse économique et devient une composante nécessaire de la théorie générale.

9. En conclusion, je ne crois pas m'abuser si j'affirme que le succès connu par l'Association de Genève au cours de ses premières dix années de vie, son expansion qu'il nous a fallu plutôt modérer que pousser, le renom dont elle jouit dans les cercles académiques dépendent autant du fait d'être née au juste moment que de l'autorité de ses membres et de l'activité méritoire de ses collaborateurs. Cela me paraissait important à souligner ici, puisque je vois dans cette correspondance avec les grandes orientations de notre époque un gage d'affirmation ultérieure de sa fonction dans les années à venir. La survie de l'assurance libre dépend de son attitude à comprendre le changement et à s'y adapter, dans l'intérêt général de l'économie. L'association de Genève se veut un instrument à ces fins. 